\title{
La formation professionnelle
}

De l'Est à l'Ouest de l'Europe : comment réussir le changement?

\section{Gisela Shaw et David Parkes}

\section{CpenEdition}

\section{Journals}

Édition électronique

URL : http://journals.openedition.org/ries/3028

DOI : 10.4000/ries.3028

ISSN : 2261-4265

Éditeur

Centre international d'études pédagogiques

Édition imprimée

Date de publication : 1 décembre 1997

Pagination : 49-62

ISSN : $1254-4590$

Référence électronique

Gisela Shaw et David Parkes, "La formation professionnelle », Revue internationale d'éducation de Sèvres [En ligne], 16 | Décembre 1997, mis en ligne le 07 juin 2013, consulté le 19 avril 2019. URL http://journals.openedition.org/ries/3028 ; DOI : 10.4000/ries.3028

Ce document a été généré automatiquement le 19 avril 2019.

(c) Tous droits réservés 


\title{
La formation professionnelle
}

De l'Est à l'Ouest de l'Europe : comment réussir le changement?

\author{
Gisela Shaw et David Parkes
}

1 Ces deux dernières décennies, presque toutes les nations d'Europe ont connu des remises en question et des transformations de leurs systèmes d'enseignement et de formation. Cela est dû, pour une grande part, aux mutations du monde du travail et à leurs conséquences sur l'emploi, les modes de travail et la technologie de production.

2 À la base de la réforme simultanée des structures de qualifications se trouvent les changements intervenus dans les processus industriels qui, selon les théories récentes de l'OCDE, doivent reposer sur une force de travail "flexible", capable de se former aux méthodes et techniques modernes de production. L'apprentissage traditionnel dotait les ouvriers des compétences nécessaires dans une industrie ou un métier donné pour toute la durée de leur vie professionnelle. Aujourd'hui, l'industrie requiert, en même temps qu'un niveau élevé de formation professionnelle, les connaissances rendant possible une éventuelle reconversion. De plus en plus, l'accent est mis sur les compétences transversales: la communication, les mathématiques appliquées, les technologies de l'information et la résolution des problèmes.

3 La présence et l'utilisation croissantes des technologies de l'information dans le processus de production transforment nombre de tâches et de profils professionnels et modifient la répartition des emplois qui diminuent dans l'industrie et augmentent dans le secteur des services. S'y ajoute un accroissement de la demande pour des travailleurs qualifiés. Paradoxalement, on constate des pénuries de main-d'œuvre qualifiée, coexistant fréquemment avec de forts taux de chômage.

4 Les effets sur les systèmes d'enseignement et de formation sont nombreux. C'est ainsi qu'il devient nécessaire :

- d'élever les taux de participation et de réussite afin qu'un plus grand nombre de travailleurs obtienne une qualification professionnelle ;

- de s'assurer que la formation professionnelle et les modes d'apprentissage répondent aux besoins de l'industrie et du commerce ; 
- de développer des systèmes qui prennent en compte et facilitent la formation continue et qui dispensent les compétences nouvelles imposées par la technologie.

Si l'ensemble des nations européennes connaît des problèmes similaires, les solutions sont, elles, relativement diverses selon des différences sociales, culturelles et politiques ; mais, malgré les divergences, des solutions communes apparaissent : développement de filières de progression; mise en place de systèmes de validation des compétences et de l'expérience antérieures ; renforcement des partenariats industrie-éducation.

Les objectifs des divers systèmes de formation professionnelle traduisent des préoccupations communes, telles que par exemple :

- réviser les qualifications générales afin qu'elles correspondent mieux aux besoins du marché ;

- développer des mesures pour aider les élèves de faible niveau et par ailleurs réduire le nombre de jeunes quittant le système sans qualification suffisante - ceci a conduit à un examen critique du contenu des cours, des conditions d'entrée, des qualifications, du financement et de l'organisation même des programmes ;

- élargir l'accès aux qualifications par une progression horizontale et verticale dans les systèmes éducatifs - ceci a exigé une plus grande ouverture des systèmes, la création de passerelles et d'équivalences, tout en éliminant les barrières inutiles ;

- adapter la formation professionnelle à l'économie de marché en Europe centrale et de l'Est.

Cependant, ainsi qu'Alison Wolf l'a relevé ${ }^{\text {: }}$

«Les États estiment qu'il est quasiment impossible de développer une politique cohérente à long terme - en grande partie parce qu'ils tentent d'atteindre des objectifs trop divers. Ils essaient de condenser au sein d'un seul système national des programmes qui impliquent des enseignements très différents - et de concilier les ambitions ou les intérêts individuels avec des préoccupations sociales au sens large, le tout dans un contexte d'incertitude face à l'avenir. »

\section{Les facteurs de changement}

\section{La réduction des ressources}

Tout au long des années quatre-vingt et quatre-vingt-dix, les systèmes d'enseignement et de formation ont connu un contexte de réduction des ressources (budgets en diminution, étudiants en plus grand nombre, moindres investissements, etc.). Les systèmes ont dû faire face en rationalisant- ce qui n'allait pas sans contradictions. Ainsi, rétrospectivement, l'introduction d'indicateurs de performance se justifiait en termes de « contrôle de qualité » mais les premiers indicateurs insistaient plutôt sur les notions de « quantités » et de diminution des coûts unitaires. Les termes mêmes utilisés ci-dessus démontrent d'ailleurs l'orientation de l'éducation vers les notions de gestion (management) et, plus particulièrement dans le monde anglo-saxon, celles du marketing.

\section{Le déplacement du niveau de décision}

9 La décentralisation (comme par exemple dans le système français) implique que la responsabilité est officiellement transférée à la région, ce qui augmente la part d'initiative locale. Ces dernières années, les nouvelles qualifications professionnelles instituées et reconnues par les partenaires sociaux ont changé la physionomie et le 
fonctionnement du système - tout en soulevant des questions quant aux rapports entre les différents acteurs. Ainsi, le regroupement d'institutions en Angleterre et au Pays de Galles (Further Education Colleges) et, dans une moindre mesure, aux Pays-Bas (collèges régionaux) ramène le pouvoir de décision au niveau des institutions mais les oblige à assumer elles-mêmes la gestion de ressources en baisse et l'enseignement à moindre coût.

\section{Le chômage des jeunes}

10 Partout en Europe, des jeunes quittent l'enseignement général ou professionnel sans diplôme ni qualification les rendant aptes au travail.

11 Les mesures prises sont restées sans effet. Dans un numéro récent du European Journal of Education $^{2}$ consacré aux nouvelles orientations dans le domaine de l'éducation et de la formation professionnelle, Damian Hanan, Ben Hôvels, Sjaak van den Berg et Michael White définissent le profil de ces jeunes ainsi que les conséquences de la sousqualification. Ils présentent les facteurs économiques ou sociaux capables d'étayer les mesures nécessaires à la restructuration de l'emploi. Ils donnent une classification des politiques éducatives possibles mais admettent qu'elles ne constituent pas une solution durable au problème des jeunes non qualifiés - problème imputable aux restructurations sociales et professionnelles.

\section{Les changements suscités par la technologie}

Les progrès des technologies en matière d'information, de communication par satellite ainsi que les médias permettent de nouvelles formules d'apprentissage de plus en plus appréciées dans le domaine de la formation continue et de l'éducation des adultes. Il est vrai que l'OCDE recommandait ces nouvelles méthodes il y a dix ou vingt ans déjà. Mais les applications pratiques étant toujours en retard d'au moins une décennie sur la conceptualisation et l'expérimentation d'un projet, les questions posées sont toujours d'actualité dans l'Europe contemporaine.

L'enseignement dit "ouvert » et l'enseignement à distance sont en général reconnus comme les mieux adaptés aux exigences modernes de l'apprentissage.

L'enseignement "ouvert » peut se définir comme étant "un système qui permet aux individus de s'inscrire aux études de leur choix, quels que soient leur lieu de résidence ou les circonstances » ou encore "l'adoption des possibilités d'apprentissage répondant aux besoins de l'élève tout en préservant son autonomie et l'efficacité des techniques d'apprentissage $»^{3}$.

Cette définition englobe une multitude de données et de variables organisationnelles telles que, par exemple, l'impact des technologies de l'information, des communications par satellite et celui des médias mais elle intègre aussi d'autres préoccupations comme l'éducation continue et l'accès des adultes à la formation et à l'enseignement.

L'enseignement « ouvert » ou à distance, qu'il concerne les études privées, les études par correspondance, à la carte ou encore les études assistées (flexistudy), permet à l'étudiant de fréquenter un centre ou une institution de façon discontinue; le système est donc bénéfique pour tous ceux qui sont en difficulté, quelle qu'en soit la raison.

Si l'enseignement à distance a surtout pour cible la population estudiantine difficilement accessible pour des raisons pratiques (comme l'éloignement géographique), 
l'enseignement « ouvert » quant à lui résout d'autres difficultés, par exemple, les lieux et horaires de travail imposés et/ou le rythme des études. Il s'ensuit des implications pédagogiques et organisationnelles pour l'enseignant qui, de "professeur ", doit plutôt devenir «conseiller» et savoir diagnostiquer, guider, travailler en équipe et dans la «transparence» et enfin - le plus important sans doute - être capable d'imaginer des outils pédagogiques soumis à évaluation publique.

Il reste difficile d'évaluer efficience et efficacité. L'efficience se mesure par une amélioration constatée soit en maintenant des indicateurs de résultats (output) à niveau constant avec moins de paramètres d'entrée (input), soit en améliorant ces indicateurs dans des proportions supérieures à celles des paramètres d'entrée. On comprend alors l'attrait de l'enseignement ouvert (et des technologies de l'éducation) s'agissant de la reconstruction des systèmes éducatifs en Europe de l'Est, centrale ou ailleurs.

Toutefois, malgré plusieurs décennies de mise en œuvre expérimentale, voire systémique, mesurer les paramètres d'entrée et les indicateurs de résultats correspondants reste une entreprise ardue - d'où l'incertitude quant à l'efficacité et au coût relatif des systèmes ouverts. Malgré tout, ces méthodes constituent (la foi aidant) une grande part de l'aide technique occidentale accordée en vue de la reconstruction des systèmes éducatifs.

Un autre danger se profile : c'est que l'avancée technologique devienne " une fin en soi ». Ce danger est réel puisque ce qui est dit ou publié sur les technologies de l'information démontre, à la quasi unanimité, que tout concept techniquement réalisable deviendra un jour réalité - même si c'est indésirable sur le plan social, politique ou technique.

\section{Les priorités politiques de l'Union européenne}

21 Les priorités politiques et les projets de la Commission européenne en matière d'éducation et de formation affectent les préoccupations immédiates des pays membres. Comme on l'a vu plus haut, les mesures actuellement mises en œuvre par l'Union européenne/la Commission sont le fruit des recherches poursuivies par l'OCDE, il y a cinq ou dix ans. C'est ainsi que le programme Leonardo de la Commission représente non pas l'approche la plus moderne, mais les usages les mieux établis.

Le Livre blanc Enseigner et apprendre - Vers la société cognitive est publié par les directions générales XXII et V, chargées de l'Éducation, de la Formation, de la Jeunesse, de l'Emploi, des Relations industrielles et des Affaires sociales. Sans grandes prétentions intellectuelles, ce document reflète pourtant avec exactitude les préoccupations européennes (et mondiales).

23 Le Livre blanc identifie trois facteurs de bouleversement qui rejoignent l'analyse présentée ci-dessus :

- l'impact de la technologie de l'information;

- l'impact de la mondialisation et de l'économie globale ;

- l'impact du progrès scientifique et technique.

24 Le document appelle à la création de nouvelles méthodes de validation des connaissances et à une approche plus souple de la certification, qui comprendrait une «carte personnelle de compétences » présentant les compétences et les savoir-faire acquis par son détenteur - en particulier quand les dites acquisitions sont évaluées en dehors du processus traditionnel d'apprentissage. 
Le Livre blanc constate que :

« Les technologies de l'information ont radicalement modifié la nature du travail et l'organisation de la production. Ces transformations ont actuellement une incidence considérable sur la société européenne ».

Il renforce les remarques de Michael Young dans une autre publication ${ }^{4}$ :

«Si on accepte la position de nombreux chercheurs, selon qui les pressions combinées des technologies modernes et de la compétition globale forceront les industries à renoncer à une organisation du travail fondée sur la division entre manuels et intellectuels - division qui n'a plus sa place face à la mondialisation alors, les distinctions traditionnelles entre enseignement général, professionnel et technique doivent s'effacer. »

Le Livre blanc plaide également en faveur d'une approche élargie du savoir permettant à l'individu d'affermir son autonomie et de s'adapter aux changements. Jarl Bengtsson, de l'OCDE, reprend cette idée ${ }^{5}$ en notant que :

« au niveau de la demande les changements sont bien plus rapides et appropriés dans les entreprises et les nations qu'ils ne le sont au niveau de l'offre. Un modèle inédit de technologie intégrée, d'organisation du travail et de formation scolaire particulièrement attentif à la flexibilité des ressources humaines semble devoir remplacer graduellement les principes tayloristes d'organisation de l'entreprise et du travail ».

Deux conclusions s'imposent : d'une part, que les structures économiques et sociales soustendent les structures de l'enseignement et leurs réformes : le changement et l'impact politique subis par les premières sont la condition nécessaire du changement dans les secondes. D'autre part, les mutations que connaissent actuellement les structures sociales et, plus particulièrement, les structures de l'économie et de l'information influenceront radicalement la nature des qualifications et des filières d'enseignement traditionnelles (jusqu'ici divisées en filières universitaire, technique et professionnelle), de même que les procédures de certification et de validation. Telles sont les bases de l'action de la Commission, quoique cette action soit parfois en avance sur la volonté des États membres d'adapter leurs structures de qualifications. Les hypothèses concernant l'impact de la mondialisation sur les filières traditionnelles pourraient de même s'avérer prématurées.

Par ailleurs, s'agissant des méthodes actuelles de développement des programmes, de certification et d'évaluation, il convient de poser la question de la continuité (dans l'avenir) de ce que nous définissons maintenant. Comme nous le remarquions plus haut, les qualifications et l'enseignement se répartissent traditionnellement en trois filières : études générales, études techniques, études professionnelles. De cette division résultent des attitudes qui vont de la parité d'estime à l'exclusion. Des études récentes analysant les raisons historiques, sociologiques, transnationales, liées au niveau social ou au marché du travail, qui justifieraient cette division (1997), démontrent que, quels que soient leurs antécédents, au plan psychologique, les populations ne peuvent entrer dans une classification aussi rigide. Les individus sont grands ou petits, bons ou mauvais élèves, mais, s'agissant d'aptitudes à l'étude ou à l'apprentissage, ils sont, avant tout, à facettes multiples. Une approche progressive ou un choix d'options étendu est probablement préférable à une séparation arbitraire des filières à un moment donné. En bref, une 
approche tantôt générale, tantôt technique, tantôt professionnelle conviendra à tel ou tel individu à différente étapes - consécutives ou non - de son développement.

Un des passages les plus intéressants du Livre blanc déclare que nous en sommes «à la fin des débats de principe ». Les extraits suivants en témoignent:

- culture générale et formation professionnelle ne sont plus des notions séparées ou opposées; l'importance des connaissances générales nécessaires à la maîtrise des compétences professionnelles est de mieux en mieux reconnue ;

- des passerelles sont jetées entre l'école et l'entreprise ;

- les barrières culturelles et idéologiques séparant l'éducation et l'entreprise disparaissent, au profit de l'une comme de l'autre ;

- selon les traditions des différents États membres, cette coopération se manifeste soit pendant la formation initiale, soit pendant la formation permanente ;

- le principe d'égalité des droits en matière d'éducation est de plus en plus appliqué dans le contexte de l'égalité des chances ;

- l'avènement des techniques d'information, ayant d'abord inquiété les professions de l'enseignement, a créé une demande pour de nouvelles formes d'éducation et de formation et commence à faire progresser l'approche pédagogique.

\section{Ce sont là d'intéressants constats...}

31 Autre question de poids pour les auteurs du Livre blanc: celle des compétences, d'une carte magnétique et d'un système ECTS de transfert d'unités de valeur (European Crédit Transfer System). Une approche basée sur la compétence (comme au Royaume-Uni) permet de définir les objectifs d'apprentissage en termes de résultats et de standards indépendants des programmes, des cours ou des méthodes de formation. Ainsi, au lieu de développer des cursus répondant à des besoins hypothétiques, des groupements professionnels représentatifs identifient les «standards professionnels » et définissent précisément les compétences requises dans les différentes branches d'activité. Les Britanniques, avec leurs «qualifications professionnelles nationales" (NVQ) ont pris un peu d'avance dans cette entreprise, mais d'autres pays de l'Union (y compris la France et l'Allemagne), en étudient actuellement la pertinence par rapport à leurs systèmes de validation des diplômes et par rapport aux programmes d'enseignement qui en résulteraient.

Le débat sur les compétences soulève nombre de problèmes. Il faut d'abord choisir une définition parmi les nombreuses possibilités offertes par différentes cultures. Il faut ensuite savoir si on définit une "compétence » comme étant une "capacité » au sens étroit, une aptitude spécifique qui peut être attestée (comme, par exemple, la capacité de plier un tuyau de métal), ou s'il s'agit de compétences «tridimensionnelles », nécessaires pour évoluer dans le temps. Enfin, mesurer ou tester des « compétences » nous permet-il d'en déduire les connaissances acquises et l'aptitude à la compréhension qui ne sont pas, elles, directement testées? Par exemple, on doit pouvoir déduire qu'une personne comprend et assimile un raisonnement par la simple observation de son comportement, tout comme on évaluerait une " compétence ".

Ce débat sur les capacités est bien illustré par une anecdote américaine des années quatre-vingt: interrogé sur le sens de sa vie, un SDF qui transporte dans un sac de plastique tous ses biens terrestres (bagperson) répond: 
« toute ma vie j'ai voulu être quelqu'un ; maintenant je sais que j'aurais dû être plus spécifique ».

\section{Les problèmes d'organisation} donc relever deux défis. système dual.

- elle s'adapte à chaque individu ; l'étudiant ; l'étudiant ;
Les nouvelles exigences de l'industrie et du commerce ont un impact sur les projections des systèmes éducatifs et, par conséquent, sur l'organisation des programmes. Il faut

En premier lieu, pour augmenter les possibilités d'accès à l'éducation et en améliorer les standards (cf. le Livre blanc), il faut d'abord faire en sorte d'établir la parité d'estime entre enseignement général et formation professionnelle. Ceci est facilité par la tendance inévitable au rapprochement entre les deux formes d'éducation, la formation professionnelle devenant de plus en plus " généraliste » dans son approche à long terme. En réponse aux mutations des structures de l'industrie, la plupart des nations réduisent le nombre de leurs spécialisations professionnelles. Ainsi, en Scandinavie, la Suède est passée de cent soixante-douze spécialisations à quatorze ou seize; la Norvège et la Finlande font de même. En Allemagne, on note une tendance identique au sein du

Par ailleurs, on s'interroge sur la modularisation. Est-elle préférable à une approche classique par matière ou par programme de formation en tant que structure d'apprentissage? En d'autres termes, la modularisation convient-elle mieux à un dispositif commun à la formation initiale et à la formation continue ainsi qu'à la validation par unités capitalisables liée à une évaluation des compétences?

La possibilité d'adapter la formation aux individus plaide en faveur de la modularisation des programmes (par opposition à l'approche par matière ou par programme); elle est donc utilisée comme méthode innovante d'aide aux étudiants en difficulté. La modularisation permet une plus grande flexibilité et des possibilités de transfert en plus grand nombre parmi les différentes filières de l'enseignement professionnel et technique - et même en dehors d'elles - une approche dite pick and mix (choisissez, mélangez).

Les principaux avantages de la modularisation peuvent être énumérés comme suit :

- elle offre un système de capitalisation et de transfert des acquis ;

- les unités capitalisables constituent un élément supplémentaire de motivation pour

- elle est souple (ou « flexible ») en termes de transfert des acquis et des compétences ;

- elle implique une réforme de la pédagogie pour promouvoir les activités ciblées sur

- et, bien sûr, elle entraîne une interaction au niveau de l'économie ;

- les modules sont facilement adaptables aux besoins locaux ou industriels ;

- les modules restent relativement indépendants des organismes institutionnels et sont adaptables à différentes formules de formation (formation de base, formation continue, formation à plein temps ou à temps partiel, à l'école ou sur le lieu de travail) ;

- enfin, la modularisation est facilement adaptable à l'enseignement ouvert.

En résumé, les partisans de l'approche modulaire plaideront la meilleure utilisation des ressources; les possibilités de choix pour l'étudiant; la flexibilité des programmes; la possibilité de varier le rythme, le moment ou le mode d'acquisition des connaissances ; la 
capacité d'être innovant; les taux de participation accrus et la meilleure utilisation des installations. Une fois adoptée par une institution, la modularisation a des conséquences organisationnelles évidentes au niveau de :

- la gestion financière ;

- la structure organisationnelle et scolaire (c'est-à-dire la façon dont l'enseignement et l'apprentissage sont organisés et financés);

- l'utilisation des ressources humaines ;

- les rapports entre l'institution et son environnement local, régional et national.

Les conséquences en ce qui concerne les directeurs d'institutions sont les suivantes :

- nouveaux critères de sélection et d'emploi pour les enseignants et les formateurs (et pour leur développement);

- mise en place d'accords permettant de créer des dispositifs d'observation, d'évaluation et de subvention des systèmes modulaires et ouverts ;

- mise à disposition de locaux, de matériel, d'équipements et autres services et adaptation des structures existantes ;

- adoption de nouvelles approches des programmes avec implications au niveau de la gestion, de la répartition des ressources, des rapports avec l'extérieur, des compétences du personnel, de la capacité d'innovation, des systèmes et structures d'organisation.

41 En revanche, une fois sorti de sa lampe, le " génie » modulaire est confronté à des tâches si amples et si complexes que très peu d'administrateurs de l'éducation ou de fonctionnaires sont à même de les comprendre ou de les maîtriser ${ }^{6}$. Les arguments en faveur de la modularisation sont tentants - mais elle pourrait mener au chaos, si, par exemple, on tient compte du nombre exponentiel d'interactions entre les diverses exigences de la gestion du temps.

\section{Études de cas : La Pologne et l'Albanie}

42 Il est intéressant de citer comme exemple certains pays d'Europe centrale, de l'Est et du Sud, qui étaient encore récemment sous des régimes socialistes à influence soviétique ; leurs systèmes éducatifs ont donc des fondements communs, même si les conditions sociales et économiques varient. Ces pays sont intéressants parce que leurs réformes se calquent souvent sur celles des Occidentaux (différant généralement selon le pays bailleur de fonds) et qu'on y retrouve donc les préoccupations de ceux-ci via, par exemple, les critères de l 'Union européenne, de la Banque mondiale ou du Conseil de l'Europe.

Par ailleurs, ces deux études de cas contiennent des différences intéressantes. La Pologne, un grand pays situé au nord de l'Europe centrale en passe de devenir membre à part entière de l'Union européenne, souffre encore sous l'impact d'incessantes mutations politiques, socio-économiques et culturelles; alors que l'Albanie, un petit pays méditerranéen subissant des pressions différentes, est animée de tout autres ambitions. En outre, les objectifs et critères de la restructuration de la formation professionnelle y sont plus larges et plus généraux que ceux de la Pologne.

\section{L'exemple polonais}

En avril 1994, le ministre polonais de l'Éducation nationale soumettait au comité Senj pour l'éducation, la science et le progrès ${ }^{7}$, un résumé de ses objectifs en matière de 
formation professionnelle. Il s'agissait de relever le niveau de l'éducation, d'élargir les profils professionnels (tout en réduisant le nombre d'options de formations), de moderniser les méthodes et le contenu de l'enseignement, enfin, de renforcer la coopération avec les partenaires sociaux (et d'obtenir leur soutien financier). Ces propositions démontraient un souci de décentralisation tout en définissant clairement les standards nationaux.

Cette évolution allait de pair avec l'importance croissante des initiatives soutenues par l'Union européenne dans le but de moderniser l'enseignement professionnel. On compte parmi ces initiatives le programme MOVE (modernisation de la formation professionnelle) dont la fonction, depuis 1992, est de promouvoir le changement dans les programmes et la politique de l'éducation. MOVE s'occupe des programmes des «lycea» et des "technika», les premiers couvrant un large secteur professionnel (y compris les études commerciales) avec une ouverture sur les études supérieures, tandis que les seconds sont plutôt orientés vers des formations spécifiques avec insertion rapide dans le monde du travail. Le programme MOVE se limite essentiellement à cinq disciplines : deux d'entre elles seront mises en œuvre prochainement, à savoir l'informatique et l'initiation au monde du travail ; trois autres doivent faire l'objet d'une modernisation : la physique, la science de l'environnement, les langues modernes.

Au printemps 1994, l'Institut européen d'éducation et de politique sociale était chargé par le groupe de travail pour la formation et les ressources humaines en Pologne (BKKK) de dresser la liste des critères et des standards requis pour établir un programme de base pour les cinq disciplines citées. Un comité d'évaluation et de standards (SEC) a été formé, comprenant cinq équipes de deux experts chacune (l'un Polonais, l'autre de nationalité étrangère). Ces équipes de deux membres (l'un « extérieur ", l'autre « local») devaient proposer des programmes modernisés selon les modèles européens.

Ces «standards européens» (le terme "européen» visant probablement les pays de l'Union) restèrent inévitablement un concept utopique et flou parce que les divers contextes nationaux, culturels et historiques ont abouti à une large gamme de systèmes différents et que, par ailleurs, les méthodes d'évaluation des résultats étaient tout aussi dissemblables d'un pays à un autre. Sans compter que des solutions convaincantes aux problèmes cruciaux affectant les systèmes "européens » d'éducation et de formation restent à trouver. Ainsi, les sociétés « européennes " persistent dans leur préférence, plus ou moins marquée, pour l'enseignement général - en tant que statut social - tout en admettant la nécessité d'une parité entre les deux formes d'éducation: générale et professionnelle. Comment parvenir à cette parité est un tout autre problème, d'autant que les attitudes de l'utilisateur, de l'institution et de la société sont déterminantes dans ce domaine.

48 Actuellement, en Pologne, les réformes en matière de formation professionnelle ont lieu dans un climat d'incertitude et d'économie fluctuante dans lequel les besoins en maind'œuvre sont, au mieux, estimés au hasard, au pire, totalement inconnus. Par ailleurs, les mutations sociopolitiques et culturelles - un préalable à toute réforme durable de l'éducation - demandent du temps et une évolution progressive des mentalités8.

Dans ce contexte complexe - mais stimulant- le comité SEC aborda sa tâche avec réalisme et prudence. Tout d'abord, en mai 1994, en accord avec le ministère de l'Éducation nationale et le BKKK, le SEC établissait les termes de références, les standards et critères d'évaluation et enfin la formule retenue pour les rapports thématiques. Un ensemble 
d'objectifs généraux et de caractéristiques applicables aux cinq domaines sélectionnés fut adopté puis adapté à chaque discipline. Ces objectifs sont les suivants :

- passage du modèle de classes traditionnelles enseignant/étudiant à un modèle d'études individuelles;

- souplesse d'organisation et amplitude du choix pour l'étudiant (modularisation);

- passage d'une approche uni disciplinaire à une approche interdisciplinaire ;

- meilleure adéquation avec les besoins du marché du travail ;

- résultats mesurables.

Il faut prendre en compte, s'agissant du dernier de ces objectifs, la tradition polonaise selon laquelle il n'est prévu aucun critère d'évaluation commun au système éducatif dans son ensemble de même que l'institution et les professeurs organisent indépendamment les examens et jugent des résultats. Si l'entrée de l'étudiant dans le système a toujours été fortement centralisée et réglementée en Pologne, le contrôle des résultats de fin d'études est, en revanche, un concept étranger au corps pédagogique polonais, ce qui interdit naturellement la mise en œuvre d'un contrôle de qualité ou la comparabilité des qualifications, ne serait-ce qu'au plan national.

51 Les véritables questions demeurent sans réponse: jusqu'où peut-on transformer un système rapidement et alors que presque tous les prérequis à cette transformation ne sont pas encore en place? Qu'est-ce qui permet d'estimer qu'un programme actualisé ne fait plus sens lorsque le contexte économique, financier, culturel et psychologique n'est pas encore stabilisé ? Questions qui ne manquent pas d'intérêt, au moment où la Pologne s'engage sur la voie de l'intégration à l'Union européenne.

\section{L'exemple albanais}

52 Prenons comme deuxième exemple un projet de l'Institut européen d'éducation et de politique sociale en Albanie. Il s'agit de faire intervenir une équipe d'experts européens pour fournir l'assistance technique nécessaire à la mise en place d'un programme de rénovation de l'éducation et de la formation professionnelle. Ce projet est particulièrement intéressant parce que les critères retenus par les experts occidentaux de diverses origines sont de portée plus générale. Ces critères sont :

- une conception plus claire de la stratégie à élaborer et la mise en place des conditions permettant sa réalisation aux niveaux régional et national;

- une structure de programmes éducatifs cohérente associant les divers types de formations (générales ou professionnelles) à leurs conséquences budgétaires ;

- une coopération efficace avec les autres ministères et les bailleurs de fonds (le ministère du Travail, par exemple) ;

- un cadre institutionnel capable de déterminer les besoins du marché et de la communauté au niveau local et de collaborer aux actions nécessaires ;

- conjointement avec les autres activités, une méthode de base pour apprécier les besoins en formation dans les divers secteurs et élaborer les programmes correspondants.

La mission que se sont donnée les experts occidentaux (allemands, néerlandais, norvégiens, portugais et britanniques) illustre parfaitement les préoccupations qui leur sont propres.

Après examen de la structure de programmes éducatifs proposée par les experts de l'Institut, le ministère de l'Éducation albanais adoptait une série de recommandations 
portant sur les caractéristiques essentielles des réformes à prévoir. Les experts étrangers et albanais (cinq de chaque côté) convinrent que la formation professionnelle du XXI ${ }^{e}$ siècle devait :

- être attrayante et permettre une progression vers les niveaux supérieurs;

- être capable de réagir aux besoins du marché du travail aux niveaux national, régional et surtout local ;

- avoir une base étendue, c'est-à-dire apporter les connaissances spécifiques à chaque occupation tout en préparant l'éventualité d'une formation continue et en aidant les jeunes à aborder leurs responsabilités de citoyens ;

- être souple dans les domaines de l'apprentissage, de l'évaluation et des certifications ;

- être efficace dans l'ouverture au plus grand nombre des plus hautes qualifications ;

- constituer un effort de collaboration dès l'instant que sont réunies en partenariat toutes les parties concernées : parents, partenaires sociaux, organismes de formation, etc. ;

- être rentable et faire un usage optimal des ressources et des équipements nécessaires à tous les niveaux de formation;

- être simple dans ses structures organisationnelles et la répartition des responsabilités.

Il est encourageant de noter que ces critères insistent sur les notions de passerelles, de contacts, de coopération et de souplesse.

Plusieurs remarques sont également applicables à toutes les nations qui reconstruisent ou réforment à l'heure actuelle leurs systèmes de formation. La première est que le changement des structures économiques et sociales est un préalable au changement des structures éducatives. Plus précisément, on estime que les mutations actuelles de l'économie et des systèmes d'information auront une influence déterminante sur les qualifications, les filières d'apprentissage et enfin sur le processus d'évaluation et de certification. Certaines expressions et locutions sont devenues incontournables dans la dialectique de l'éducation: par exemple, flexibilité (ou souplesse), compétences de base, introduction au monde du travail, accréditation, qualité, égalité, indicateurs de performance et même marketing.

En second lieu, le passage de la formation initiale à la formation continue est devenu moins crucial que l'intégration des deux phases dans un même cursus. Si les États membres de l'Union sont individuellement «jaloux» de l'intégrité de leur système national, certaines convergences entre eux manifestent une réponse identique à des problèmes communs. On constate une résistance à l'« harmonisation » bureaucratique émanant de Bruxelles mais on admet la nécessité grandissante de la "transparence » entre les systèmes, telle qu'elle pourrait survenir par la coopération entre États.

59 Par ailleurs, un accent particulier est mis sur l'importance du management ou " gestion » de l'éducation, tant conceptuellement qu'en termes de réalisation, avec une prédominance du jargon anglo-saxon du management.

60 Il semble enfin qu'on souhaite l'émergence, dans les pays de l'Est, d'un système représentant le nec plus ultra (quoi qu'on veuille entendre par là) de l'éducation selon les critères de l'Union et de l'OCDE, et permettant de réduire la centralisation et de transférer les responsabilités au niveau régional.

61 Les deux études de cas ci-dessus évoquent d'autres questions de grand intérêt. 
62

, celui du délai nécessaire pour accomplir une restructuration raisonnable d'un système d'éducation et de formation, depuis la conception des stratégies jusqu'à leur application. Alors qu'en Occident, une réforme isolée peut prendre six à huit ans pour se mettre en place, les pays d'Europe centrale et de l'Est n'ont eu jusqu'ici que huit ans pour revoir leurs systèmes en totalité.

63

\section{NOTES}

1. Alison Wolf, "Vocational Education Policy in a European Context», European Journal of Education, 1993, vol. 28, $\mathrm{n}^{\circ} 2$.

2. . Hannan, B. Hovels, S. Van den Berg et M. White, " "Early leavers" from Education and Training in Ireland, the Netherlands and the United Kingdom », European Journal of Education, Recent Trends in Vocational Education and Training, 1995, vol. 30, n 3.

3. David Parkes, « Managing the Gaps : the Interpretation of Policy to Create a Second Chance in the Further Education Systems of the UK », in : Second Chance Education, Ed Inbar, Falmer Press, 1990.

4. Michael Young, "Bridging the Academic/Vocational divide: Two Nordic Case Studies", European Journal of Education, 1993, vol. 28, $\mathrm{n}^{\circ} 2$.

5. J. Bengtsson, «Labour Markets of the Future: the Challenge to Education Policy Makers", European Journal of Education, vol. 28, $\mathrm{n}^{\circ} 2$.

6. David Parkes, Tripartism and Other Divisions in Post-Compulsory Education, U.K., Further Education Development Agency, 1997.

7. P. Grootings, The Vocational Educational and Training System in Poland, Unpublished draft manuscript, 1994.

8. G. Shaw, «Modernising Curricula in Vocational Education and Training in Poland: a Case Study », European Journal of Education, 1995, vol. 30, n 3.

\section{RÉSUMÉS}

Relayant et amplifiant les recherches de l'OCDE, la Commission européenne fait de la formation tout au long de la vie une priorité politique. Les auteurs proposent de porter un regard critique sur le Livre blanc "Enseigner et apprendre - Vers la société cognitive». S’ils reprennent à leur compte l'inventaire des causes de mutation, réaffirment nettement la diversité des transferts entre culture générale et formation professionnelle et valorisent plusieurs dispositifs de 
formation, ils rappellent par une étude de cas, conduite sur deux pays d'Europe centrale, la Pologne et l'Albanie, que les orientations prônées n'ont d'impact que sur des systèmes sociaux répondant à une série de pré-requis d'ordre économique, financier, culturel et que ces pré-requis n'offrent de garantie qu'après une remise en cause totale de l'existant.

INDEX

Index géographique : Europe, Pologne, Albanie

Mots-clés : formation tout au long de la vie, politique européenne, formation professionnelle, politique éducative

\section{AUTEURS}

\section{GISELA SHAW}

Directeur de recherches. Faculty of European Studies, University of the West of England, Bristol, Grande-Bretagne

\section{DAVID PARKES}

Deputy Director, Institut européen d'éducation et de politique sociale, université de ParisDauphine, Paris, France 